\title{
THE EFFECTS OF SOCIODEMOGRAPHIC FACTORS FAMILY SUPPORT AND LOCAL CULTURE ON THE USE OF LONG TERM CONTRACEPTIVE IN TULUNGAGUNG EAST JAVA
}

\author{
Dika Yanuar Frafitasari',2), Uki Retno Budihastuti3), Bhisma Murti²) \\ 1)Institute of Health Sciences Bhakti Wiyata, Kediri \\ ${ }^{2)}$ Masters Program in Public Health, Universitas Sebelas Maret \\ 3)Department of Obstetrics and Gynecology, Dr. Moewardi Hospital, Surakarta
}

\begin{abstract}
Background: Family planning is a cost-effective strategy for reducing maternal and child morbidity and mortality. However, sociocultural expectations and values attached to marriage among women of reproductive age remain an impediment to using family planning method. The purpose of this study was to investigate the effects of sociodemographic factors, family support, and local culture, on the use of long term contraceptive in Tulungagung, East Java.

Subjects and Method: A cross sectional study was carried out at 25 community health posts (posyandu) in Kauman, Tulungagung, East Java, in January 2019. A sample of 200 women of reproductive age was selected by stratified random sampling. The dependent variable was long-term contraceptive method use. The independent variables were age, education, knowledge, employment, husband support, women's perception, local culture, and posyandu. The data were collected by questionnaire and analyzed by a multiple logistic regression.

Results: Age $\leq 35$ years old $(\mathrm{OR}=5.43 ; 95 \% \mathrm{CI}=1.91$ to $15.42 ; \mathrm{p}=0.001)$, education $\geq$ senior high school $(\mathrm{OR}=6.42 ; 95 \% \mathrm{CI}=2.33$ to $17.65 ; \mathrm{p}<0.001)$, high knowledge $(\mathrm{OR}=5.71 ; 95 \% \mathrm{CI}=1.94$ to $16.76 ; \mathrm{p}=0.001)$, working outside the house $(\mathrm{OR}=5.08 ; 95 \%$ $\mathrm{CI}=1.88$ to $13.68 ; \mathrm{p}=0.001)$, high family support $(\mathrm{OR}=6.50 ; 95 \% \mathrm{CI}=2.32$ to 18.18 ; $\mathrm{p}<0.001)$, positive women's perception $(\mathrm{OR}=5.56 ; 95 \% \mathrm{CI}=2.05$ to $15.06 ; \mathrm{p}=0.001)$, and supportive local culture $(\mathrm{OR}=4.56 ; 95 \% \mathrm{CI}=1.79$ to $11.60 ; \mathrm{p}=0.001)$ increased long term contraceptive method use. Posyandu increased long term contraceptive use but it was statistically non-significant $(\mathrm{OR}=2.01 ; 95 \% \mathrm{CI}=0.83$ to $4.88 ; \mathrm{p}=0.121)$.

Conclusion: Age $\leq 35$ years old, education $\geq$ senior high school, high knowledge, working outside the house, high family support, positive women's perception, and supportive local culture increase long term contraceptive method use. Posyandu increases long term contraceptive method use but it was statistically non-significant.
\end{abstract}

Keywords: long term contraceptive, family planning, family support, social culture

\section{Correspondence:}

Dika Yanuar Frafitasari. Masters Program in Public Health, Universitas Sebelas Maret. Jl. Ir. Sutami 36A, Surakarta 57126, Central Java, Indonesia. Email: dikayanuar@gmail.com. Mobile: +628563430126 .

The $6^{\text {th }}$ International Conference on Public Health Best Western Premier Hotel, Solo, Indonesia, October 23-24, 2019 | 169 